\title{
tic\&société
}

Vol. 10, N 2-3 | 2ème semestre 2016 - 1er semestre 2017

Spécial varia

\section{La construction d'une identité numérique professionnelle chez les « digital natives » : fiction ou réalité?}

\section{Maud PÉLISSIER}

\section{(2) OpenEdition}

\section{Journals}

Édition électronique

URL : http://journals.openedition.org/ticetsociete/2077

DOI : 10.4000/ticetsociete. 2077

\section{Éditeur}

Association ARTIC

\section{Édition imprimée}

Pagination : 71-97

\section{Référence électronique}

Maud PÉLISSIER, «La construction d'une identité numérique professionnelle chez les « digital natives » : fiction ou réalité ? », tic\&société [En ligne], Vol. 10, № 2-3 | 2ème semestre 2016 - 1er semestre 2017, mis en ligne le 30 avril 2017, consulté le 17 juin 2020. URL : http://

journals.openedition.org/ticetsociete/2077; DOI : https://doi.org/10.4000/ticetsociete.2077 
tic\&société - 10(2-3), 2016-2017

\title{
La construction d'une identité numérique professionnelle chez les « digital natives » : fiction ou réalité ?
}

\begin{abstract}
Maud PÉLISSIER
Maud Pélissier est maître de conférences au laboratoire de Sciences de l'information et de la communication I3M/IMSIC de l'université de Toulon. Ses travaux de recherche portent sur la culture numérique et les dynamiques d'appropriation des technologies digitales dans le contexte des organisations de connaissance et des institutions culturelles. Économiste de formation, elle s'intéresse aussi aux transformations actuelles des industries culturelles sur le plan des activités de création, de médiation et de réception au travers de la question des communs informationnels. maud.pelissier@univ-tln.fr
\end{abstract}




\section{La construction d'une identité numérique professionnelle chez les « digital natives » : fiction ou réalité ?}

Résumé : Aujourd'hui, il existe une pression de plus en plus forte en provenance des concepteurs des réseaux socionumériques professionnels, mais aussi des acteurs de l'éducation, comme ceux du monde professionnel, pour inciter les jeunes à mettre en œuvre une identité numérique professionnelle, considérant cette démarche comme un facteur déterminant dans la réussite de leur trajectoire professionnelle. Partant des résultats d'une enquête quantitative réalisée auprès d'un échantillon de jeunes âgés de 16 à 24 ans, nous proposons de mettre en évidence les usages informationnels et communicationnels accompagnant leurs choix en matière d'orientation et d'insertion professionnelles afin d'évaluer s'ils traduisent une stratégie d'élaboration d'une identité numérique professionnelle.

Mots-clés : identité numérique professionnelle, usages, jeunes, réseaux socionumériques.

Abstract: It is now well documented that young generations, having an intensive use of digital technologies especially in entertainment contexts, build digital identities that can take different shapes depending on their goals. This article aims to study how these young generations mobilize their skills and daily digital uses to build a professional digital identity. This question is significant because both educational and economic areas urge them to do so. This analysis bases its argument on an empirical investigation focusing on the digital uses of the youth (from 16 to 24 years old) in building their professional trajectories.

Keywords: digital identity, professional trajectory, digital social network.

Resumen: Hoy en día existe una triple presión, cada vez mayor, procedente de los creadores de las redes socionuméricas profesionales, de los agentes educativos y del mundo profesional; presión que incita a los jóvenes profesionales a crear y mantener una identidad profesional digital, de forma que dicha identidad se convierte en un factor determinante de su trayectoria profesional. A partir de los resultados de una encuesta cualitativa, realizada en una muestra de jóvenes de 16 a 24 años, se propone mostrar los 
La construction d'une identité numérique professionnelle chez les « digitales natives » : fiction ou réalité ?

usos relacionados con la información y con la comunicación que acompañan sus decisiones en materia de orientación y de inserción profesionales, con el fin de evaluar si dichos usos reflejan una estrategia de elaboración de una identidad digital profesional.

Palabras clave: identidad digital profesional, usos, jóvenes, redes socio- digitales. 


\section{Maude PÉLISSIER}

\section{Introduction}

L'environnement numérique a donné naissance à de nouveaux modes de communication et d'échange de l'information qui s'insèrent de façon croissante dans les activités quotidiennes. De ces espaces de négociation entre les techniques et les pratiques, notre perception du temps et de l'espace, notre relation aux autres, à soi s'en trouvent affectés (Doueihi, 2008). En particulier, le processus de construction identitaire a trouvé dans les réseaux socionumériques ${ }^{1}$ (RSN) des espaces privilégiés pour se déployer, comme l'a très bien montré le sociologique Dominique Cardon (2009). Ces espaces sont devenus le creuset de ces métamorphoses au cours desquelles une identité d'un nouveau genre est en train d'éclore. Les jeunes générations - les fameux digital natives (entendus ici comme ceux étant nés avec l'émergence de l'internet, dans les années 1990) - s'avèrent particulièrement adeptes de ce type d'environnement, qui leur offre un espace de communication interpersonnelle ludique et inédit au sein duquel elles peuvent se forger à l'envie une identité dite "numérique », en jouant avec les multiples registres disponibles de design de la visibilité. Florian Dauphin (2012) souligne que différents travaux mettent en évidence le lien entre les usages des TIC et la construction identitaire des jeunes. Pour lui, "l'observation des pratiques juvéniles des blogs et des réseaux sociaux montre que le mot d'ordre de cette construction identitaire numérique est de ne pas rester invisible et non identifiable dans la masse » (2012, parag. 12).

Durant le passage à la vie adulte, les usages en ligne évoluent, s'élargissent au-delà des fonctions de sociabilité et de loisirs privilégiées jusqu'alors. Les jeunes adultes sont progressivement amenés à consommer, à apprendre, à travailler, à gérer leur vie administrative, voire leur engagement citoyen au sein de l'environnement numérique. Ils sont aussi et surtout de plus en plus incités, dans un contexte socioéconomique de valorisation croissante de la compétition individuelle, à une mise en scène de soi professionnelle inédite, à construire un discours sur eux-mêmes, leurs

\footnotetext{
${ }^{1}$ Nous faisons ici référence à la définition de Stenger et Coutant (2010) : « Les RSN constituent des services Web qui permettent aux individus : (1) de construire un profil public ou semi-public au sein d'un système, (2) de gérer une liste des utilisateurs avec lesquels ils partagent un lien, (3) de voir et naviguer sur leur liste de liens et sur ceux établis par les autres au sein du système et (4) [de fonder] leur attractivité essentiellement sur les trois premiers points et non sur une activité particulière » (p. 221).
} 
La construction d'une identité numérique professionnelle chez les « digitales natives » : fiction ou réalité ?

« compétences », leurs projets, leurs réalisations passées et leurs perspectives futures, comme le défend María Eugenia Longo (2011) dans un rapport de l'INJEP intitulé " Transitions des jeunes vers la vie adulte : processus d'insertion et rapports à la vie professionnelle ॥. Cette mise en scène de soi professionnelle est enseignée au travers de programmes éducatifs dans les enceintes du lycée comme de l'université et s'appuie sur des dispositifs sociotechniques divers, comme l'ePortofolio ou les portefeuilles d'expérience et de compétences. L'environnement numérique a aussi vu naître des plateformes relationnelles, telles Viadeo et Linkedln, présentées comme des leviers désormais indispensables dans l'élaboration de la trajectoire professionnelle. Tel est, en particulier, le message véhiculé par leurs concepteurs, non seulement auprès de leur cible prioritaire, les actifs, mais aussi, désormais, auprès des plus jeunes. Linkedln a ainsi annoncé, en septembre 2013, vouloir baisser de 18 à 13 ans l'âge minimal requis pour ouvrir un profil, car, comme le dit Laurence Bret, la directrice marketing: "Les adolescents s'intéressent de plus en plus tôt à leur avenir et nous pensons que le réseau est au cœur de la réussite professionnelle, les gens qui sont bien connectés ont plus de chances de réussir » (citée dans Lelong, 2013, parag. 2).

Face à cet univers de prescription forte en provenance d'horizons divers incitant les jeunes à mettre en place une stratégie communicationnelle de mise en valeur de leur trajectoire professionnelle (allant de l'orientation à l'insertion professionnelle), nous jugeons utile d'étudier quels sont leurs usages réels et d'évaluer s'ils construisent leur identité numérique professionnelle en suivant les prescriptions sociotechniques évoquées. Nous prendrons appui sur les résultats d'une étude quantitative réalisée préalablement auprès de jeunes âgés de 16 à 24 ans de la région ProvenceAlpes-Côte d'Azur par l'Observatoire des TIC (Pélissier et Pérocheau, 2013). Cette enquête nous permettra de révéler les usages informationnels et communicationnels accompagnant leurs choix en matière d'orientation et d'insertion professionnelles afin d'évaluer s'ils traduisent une stratégie d'élaboration d'une identité numérique professionnelle. La question de la construction d'une identité numérique professionnelle chez les jeunes est un domaine de recherche balbutiant en sciences de l'information et de la communication. Mentionnons toutefois les travaux pionniers de Julien Mésangeau (2012) qui nous serviront de référence. La notion d'identité numérique professionnelle occupant une place centrale dans notre étude, la première partie de cet article vise à mieux circonscrire, d'un point de vue théorique, cette notion. La méthodologie de l'enquête, les résultats de notre étude 
empirique et une mise en discussion feront l'objet d'une seconde partie.

\section{L'identité numérique professionnelle: un essai de conceptualisation}

L'expression « identité numérique professionnelle " (INP) est une notion théorique aux contours encore flous et qui a d'abord circulé dans les milieux professionnels avant de devenir un objet de recherche dans le champ des sciences sociales. Ainsi, il n'existe pas, à ce jour, de consensus sur ce concept en devenir. L'objet de cette première partie est d'esquisser des pistes de réflexion permettant de circonscrire cette notion. Nous proposons comme socle théorique initial les travaux du sociologue Dominique Cardon sur l'identité numérique (2009) et le design de visibilité des plateformes Web 2.0 (2008). Nous formulerons l'hypothèse que l'INP résulte d'un processus de coconstruction, de négociation entre le design des RSN professionnels et les intentionnalités des usagers.

\subsection{Un processus de création de son moi professionnel sur l'espace numérique}

Dominique Cardon a étudié les conséquences de cette dynamique expressiviste (Allard, 2007) qui se manifeste de façon croissante au travers des réseaux socionumériques (ou des plateformes relationnelles) en termes de construction identitaire. Les RSN offrent des espaces de représentation et de projection de soi permettant le dépôt de signes relationnels divers. En accord avec une approche sociotechnique, le sociologue propose de définir l'IN comme le résultat d'un processus de coconstruction entre «les instructions des interfaces d'enregistrement et les calculs que font les utilisateurs pour produire la meilleure impression d'euxmêmes ", autrement dit entre "les stratégies des plateformes et les tactiques des utilisateurs " (Cardon, 2008, p. 98). II précise que cette dynamique de construction identitaire résulte elle-même "d'un double mouvement d'extériorisation de soi dans des signes et de réglage réflexif de la distance à soi » (ibid., p. 98). L'extériorisation de soi correspond à un processus de subjectivation, un "processus de création continue de soi » (ibid., p. 100), et s'exprime au travers de différents signes déposés sur les RSN permettant de signifier sa singularité. La seconde dynamique correspond à un processus intentionnel de simulation visant à jouer sur la distance entre son identité réelle et son identité numérique. Les plateformes relationnelles offrent 
La construction d'une identité numérique professionnelle chez les « digitales natives » : fiction ou réalité ?

à cet égard à l'utilisateur « un pouvoir renforcé pour styliser et modifier leurs apparences jusqu'à menacer l'authenticité des définitions de soi » (ibid., p. 102).

L'identité numérique professionnelle participe d'une logique analogue. Nous proposons de la définir comme une stratégie relationnelle de projection de sa trajectoire professionnelle dans les RSN. Ceux-ci constituent des espaces privilégiés pour déposer des indices de ce processus de construction de son moi professionnel en offrant un espace dédié à la publicisation de sa trajectoire professionnelle. Le processus d'extériorisation va ainsi consister en l'exhibition de signes témoignant de son "identité incorporée " professionnelle, civile et sociale, comme le diplôme, l'université ou l'école fréquentée, mais aussi de signes renvoyant à son " identité agissante », comme l'affichage de son réseau relationnel professionnel ou encore de traits permettant de signaler ses activités et révélant "des compétences incorporées, des savoir-faire et des qualités mises en œuvre pour la réalisation de telle ou telle activité » (Cardon, 2008, p. 101). La réalisation d'activités bénévoles et la mise en ligne d'un mémoire de stage en sont des exemples parmi d'autres.

Le processus de construction de son moi professionnel se manifeste également par une dynamique de simulation correspondant à un « processus de rationalisation réflexive du rapport à l'autre " (Cardon, 2008, p. 100). Les RSN offrent en effet la possibilité de "composer " avec son identité et de modeler et de parfaire son image en accentuant certains traits et en en effaçant certains autres. Cette dynamique de simulation peut répondre à des motivations diverses et singulières d'un individu à un autre et prendre des formes multiples. Dans le cadre du processus de construction de l'INP, il ne s'agit plus de se dissimuler sous des pseudos, de naviguer entre des identités multiples, mais de construire une scénarisation de son moi professionnel le plus en adéquation avec les attentes de futurs employeurs. Le défi est de communiquer sous son meilleur jour tous les indices constitutifs de sa trajectoire professionnelle. Comme le soulignent à juste titre Valérie Larroche et Jacqueline Bérard (2016),

"l'identité comporte aussi une adaptation du discours identitaire aux codes de l'univers professionnel visé. [...] II s'agit entre autres de traduire certains traits identitaires obtenus dans la communauté étudiante, pour les rendre signifiants auprès des professionnels. Faire part de ses capacités d'adaptabilité et d'autonomie $[\ldots]$ est un argument positif » (p. 13).

tic\&société - 10(2-3), 2016-2017 


\section{Maude PÉLISSIER}

Cette dynamique peut ainsi conduire à des cas extrêmes de dissimulation de certains éléments de sa trajectoire professionnelle jugés embarrassants ou, à l'inverse, à en exagérer certains autres.

De leur côté, les RSN professionnels favorisent un rapport communicationnel à l'autre envisagé comme une relation stratégique, ainsi que le soulignent Julien Mésangeau et Arnaud Povéda (2013): "L'étude des documentations accompagnant les usages des RSP incite à un exercice réflexif où l'usager doit s'apparier sur un mode optimisé » (p. 125). Rappelons que ces RSN professionnels ont pour objectif ultime d'optimiser l'employabilité de leurs usagers au travers de pratiques de réseautage visant à choisir de façon rationnelle les différents appariements proposés ou suggérés. Sans le dire, ils remettent au goût du jour, en l'adaptant à l'environnement numérique, l'un des résultats empiriques majeurs de l'article sur la "force des liens faibles " du sociologue Mark Granovetter (1973). Ce dernier avait en effet montré que la structure du réseau social ${ }^{2}$ d'un individu est un déterminant essentiel dans l'obtention d'un emploi (Mercklé, 2011). Les concepteurs des RSNP avancent un argument similaire en invoquant tout le potentiel de leurs dispositifs: ils offrent non seulement la possibilité de "construire " son propre réseau social et de le transformer en véritable capital social, mais aussi de pouvoir l'utiliser dans une optique stratégique ${ }^{3}$. Les dynamiques de construction de l'IN comme de l'INP se déploient ainsi au sein de plateformes relationnelles dont le design est susceptible d'exercer une force prescriptive en matière comportementale.

Notons enfin que ce processus de mise en visibilité de son moi professionnel s'accompagne aussi de dispositifs sociotechniques dédiés, proposés de façon complémentaire pour favoriser la construction de son INP. On peut citer l'ePortfolio ${ }^{4}$ ou bien encore le portefeuille d'expériences et de

\footnotetext{
${ }^{2}$ Le réseau social est ici défini comme l'ensemble des individus qui lui sont reliés par des chaînes d'interconnexion plus ou moins longues. Ces chaînes interindividuelles traduisent des liens forts (relations soutenues et fréquentes) ou faibles (simples connaissances).

${ }^{3}$ Notons que les concepteurs de ces dispositifs numériques omettent volontairement de souligner que le réseau social construit sur un RSN professionnel et celui forgé dans sa vie de tous les jours ne sont pas nécessairement les mêmes.

${ }^{4}$ Voir « Innovations numériques dans le supérieur. ePortefolio : un dispositif national » sur Le portail du numérique dans l'enseignement supérieur du gouvernement français : <www.sup-numerique.gouv.fr/cid94605/eportfolio-undispositif-national.html>, dernière consultation le 19 mars 2017.
} 
La construction d'une identité numérique professionnelle chez les « digitales natives » : fiction ou réalité ?

compétences (PEC) $)^{5}$ mis à la disposition d'un nombre croissant de jeunes étudiants pour les accompaqner dans un travail d'identification de leurs compétences, de repérage de leurs atouts et de mise en valeur de leur parcours de formation et de leurs expériences professionnelles. Les slogans « Je fais mon bilan » et " J'aqis et ie communique » attestent à cet éqard de l'importance croissante de la dimension communicationnelle dans la construction de leur trajectoire professionnelle.

\subsection{Identité et réputation: deux facettes d'une même réalité}

L'environnement numérique n'est pas un «lieu » avec des frontières délimitant clairement les espaces publics des espaces privés, les espaces de loisirs des espaces professionnels. Il a même donné naissance à des espaces d'un nouveau genre dans lesquels la frontière privée/publique est modulable (Cardon, 2008). Par ailleurs, tous les signes, les messages, les documents accompagnant les activités communicationnelles et informationnelles dans cet espace sont assimilables à des traces qui ne s'effacent pas (fonction mémorielle du Web) et relativement accessibles, justement en raison de cette plasticité complexe sur le plan de la visibilité. Autrement dit, l'environnement numérique dans lequel se forge et se construit l'identité laisse apparaître une autre facette de ce phénomène. Pour Olivier Ertzscheid (2009), l'homme devient un document comme un autre et l'identité numérique se réduit à une

" collection des traces (écrits, contenus audio ou vidéo, messages sur des forums, identifiants de connexion, actes d'achat ou de consultation...) que nous laissons derrière nous, consciemment ou inconsciemment, au fil de nos navigations [sur le réseau] et de nos échanges. Une fois "remixées" par les moteurs de recherche ou les sites de réseaux sociaux, ces traces définissent le périmètre de notre réputation numérique » (p. 54).

L'identité perd ici toute son humanité pour se transformer en une entité extériorisée qui «[désagrège] la personne et

\footnotetext{
${ }^{5}$ Voir la page du consortium PEC (Portefeuille d'Expériences et de Compétences) : $<$ www.pec-univ.fr/accueil-pec-11496736.kjsp>, dernière consultation le 19 mars 2017.
} 
[mobilise] du même coup des aspirations à maîtriser son identité » (Merzeau, 2009, p. 79). Construire son identité numérique est un processus qui laisse des marques, visibles par tous. Néanmoins, étant devenues visibles, indexables, calculables, celles-ci engagent plus que jamais la réputation entendue comme " la valeur informationnelle de nos actions, la trace de notre conduite que nous laissons inévitablement dans les opinions des autres à chaque fois que nous interagissons avec le monde social, et qui oriente leurs actions vis-à-vis de nous » (Origgi, 2007, p. 103).

Cette aspiration à " maîtriser » son identité en cachant ce que l'on ne veut pas laisser voir et, inversement, à valoriser ce que l'on juge bon d'être vu en fonction des objectifs poursuivis donne lieu à des stratégies communicationnelles d'un nouveau genre dont le but est d'optimiser sa réputation en ligne. Pour Samy Ben Amor et Lucia Granget (2011), la personne "se trouve désormais au cœur d'une démarche managériale pour la construction d'une image et la protection de son identité ॥ (p. 109). Cette démarche consiste à mettre en œuvre des stratégies de communication, dans l'environnement numérique, visant à valoriser sa réputation, selon une logique de personal branding. Les personnes publiques, comme les artistes ou les hommes politiques, ont été les premiers à comprendre l'intérêt de maîtriser leur image dans l'environnement numérique, car se jouaient en amont leur respectabilité et leur notoriété, éléments essentiels de leur réputation et in fine de leur "valeur économique ». JeanClaude Domenget (2013) emploie l'expression de « professionnels de la visibilité » pour qualifier ces nouvelles stratégies d'e-réputation utilisées à différentes échelles par les usagers du Web. Pour Camille Alloing et Julien Pierre (2012), la réputation en ligne est à la fois signe (donnée et information) et relation (interaction et contexte) et s'inscrit dans une rationalisation de l'agir. Reprenant ainsi la définition de Max Weber, les auteurs précisent que « [l]es possibilités de calcul et de capture vont alors servir les besoins économiques de réduction d'incertitude et de maximisation de son capital social (Origgi, 2007, p. 5), et c'est une industrialisation de la réputation qui va faire son nid au sein de l'économie sociale » (p. 6).

Si, pour les acteurs de la sphère publique, la maîtrise de leur capital réputationnel est essentielle, il n'est pas pour autant évident que cette logique de personal branding soit appropriée par la majorité des usagers du Web. Selon Dominique Cardon (2008), alors même que les plateformes relationnelles constituent des espaces de grande exposition, il est « frappant de constater, en contraste avec toutes les inquiétudes relatives 
La construction d'une identité numérique professionnelle chez les « digitales natives » : fiction ou réalité ?

à la surveillance numérique et au respect de la privacy, que les usagers prennent beaucoup de risques avec leur identité » (p. 97). Plus récemment, une étude réalisée aux États-Unis par une chercheuse de l'université de l'Ohio, Elizabeth Stoycheff (2016), spécialiste des médias, montre à l'inverse que la surveillance croissante sur le Web engendre une culture d'autocensure, menaçant ainsi l'expression des opinions minoritaires et contribuant à renforcer l'opinion dominante.

Quels sont les enseignements que l'on peut tirer de ces observations dans le cadre de l'identité numérique professionnelle? Au même titre que pour les personnes publiques, il apparaît assez évident que construire son INP implique aussi et simultanément de prendre en compte cette dimension réputationnelle, et ce, d'autant que la perméabilité entre tous les espaces numériques d'expression de son identité, privée et professionnelle, peut conduire à un " mélange des genres » nuisible à la réputation de la personne. De nombreux exemples ont révélé de quelle manière des traces de l'identité privée rendues visibles dans des espaces publics ou en clair-obscur ont pu être utilisées, à l'insu des personnes concernées, par les acteurs du monde professionnel (justifiant ainsi un licenciement ou un refus d'embauche). Pour la jurisprudence en France, les différents arrêtés tendent à considérer Facebook comme étant un espace public où l'on renonce à une certaine discrétion ${ }^{6}$. Cela devrait donc se traduire par une stratégie visant à maîtriser tous les flux informationnels et communicationnels laissés comme autant de traces multiples sur le Web, mais aussi par une stratégie dont l'objectif est d'optimiser l'utilisation des RSN au travers de la mise en place de stratégies de réseautage. En effet, il ne s'agit pas seulement d'être inscrit sur un RSN, encore faut-il élaborer des stratégies qui parferont son capital social, car une réputation professionnelle se mesure aussi à l'aune de sa capacité à gérer son réseau social numérique.

\subsection{Les enseignements des premières études sur les usages des RSNP}

Dans le champ des sciences de l'information et de la communication, à ce jour, rares sont les études empiriques qui ont pris pour objet d'étude l'identité numérique professionnelle. Le travail de thèse de Julien Mésangeau (2012) mérite toutefois

\footnotetext{
${ }^{6}$ Voir CHAUDEAU, C., 2009, « Facebook et licenciement : les statuts qui les ont fait virer », Cadremploi, 13 septembre, <www.cadremploi.fr/editorial/conseils/droit-dutravail/detail/article/facebook-les-statuts-qui-les-ont-fait-virer-ou-pas.html>, dernière consultation le 19 mars 2017.
} 
d'être cité, car il a ouvert la voie à cette question en livrant une analyse sur une thématique plus circonscrite mais connexe, relative aux « pratiques du réseautage en ligne " sur le réseau socionumérique professionnel Linkedln. Une étude empirique portant sur un groupe d'anciens étudiants issus d'une grande école (Sciences Po Paris Alumni [SPPA]) en est le point de départ. Ce travail a ainsi permis à Julien Mésangeau de dresser une typologie sur les pratiques de réseautage en ligne et de montrer qu'il s'agit d'un phénomène pluriel, se manifestant au travers de trois formes singulières: le netmining, qui désigne les pratiques visant à cumuler des contacts et qui repose « sur le pari que tout utilisateur, dans une certaine limite, peut [...] offrir une opportunité potentielle » (Mésangeau, 2012, p. 286) ; le networking, employé pour qualifier les «pratiques de sélection des relations qui reposent sur une forme de rationalité instrumentale » (ibid.), car chaque contact est sélectionné pour une raison particulière ; enfin, le netstiking, qui « se réfère aux pratiques de mise en relation où l'utilisateur reconstitue sur Linkedln un environnement social familier » (ibid., p. 151), « un cercle de proches » (ibid., p. 229).

L'autre apport de son enquête est de s'interroger sur les déterminants de ces pratiques de réseautage. Plusieurs résultats sont à mentionner. En premier lieu, il révèle que, contrairement à ce que l'on aurait pu croire, tous ces acteurs n'agissent pas selon une action planifiée. Ils n'ont pas une " exploitation économiquement rationnelle » de cet outil impliquant une gestion stratégique des relations sociales, comme il l'avait supposé au départ: "Nous pensions avoir affaire à des pratiques stratégiques, où le choix des contacts repose invariablement sur un calcul rigoureux consistant à évaluer l'utilité de chaque relation invitée en ligne. En réalité, les pratiques identifiées se sont avérées beaucoup plus nuancées ॥ (Mésangeau, 2012, p. 32) et souvent en décalage par rapport aux prescriptions d'usage. Telle est une des conclusions principales de la thèse de Julien Mésangeau. Pour autant, cela ne signifie pas que les pratiques de ces acteurs sont dénuées de toute forme de rationalité, qui peut exister sous des formes assouplies et moins exigeantes. En s'appuyant sur les écrits d'Emmanuel Lazega, il défend l'idée que la rationalisation de l'échange social ne relève pas nécessairement d'un calcul économique, à visée utilitaire, mais peut aussi avoir une visée symbolique: "II existe différentes formes de rationalité dans les pratiques de mise en relation des utilisateurs » (ibid., p. 216).

Dans un article ultérieur écrit avec Arnaud Povéda (2013), la question des déterminants de ces pratiques de réseautage en ligne est complétée par une approche 
La construction d'une identité numérique professionnelle chez les « digitales natives » : fiction ou réalité ?

sociotechnique, cadre d'analyse par ailleurs largement mobilisé dans les travaux sur l'analyse des usages des dispositifs sociotechniques (Coutant, 2015). L'apport majeur de cette approche est d'insister sur la double médiation, technique et sociale, comme l'avait défini originellement Josianne Jouët (1993), que Julien Mésangeau et Arnaud Povéda réinterprètent en ces termes: " si la plastique du médium définit l'espace des possibles offerts à la socialisation de l'outil de communication, l'acte communicationnel va quant à lui contribuer à construire le sens que l'utilisateur attribue à ses actes " (Povéda, 2013, p. 184). Le RSP défini comme un artefact communicationnel, ou bien encore comme un outil médiatisant un espace de relations, impose un cadre de négociation avec les utilisateurs. Ces derniers doivent composer non seulement avec les spécificités techniques de celui-ci, mais aussi avec la dimension normative de l'artefact proposant un cadre d'interactions prescrit. Or, dans le même temps, ces mêmes usagers utilisent cet artefact en fonction de leur propre subjectivité, elle-même en partie déterminée par «leurs habiletés sociales et techniques » (ibid., p. 186).

Ainsi, au travers d'une enquête d'observation en ligne portant sur mille profils d'utilisateurs, ils montrent que, suivant les formations suivies et les métiers envisagés, les acteurs ne valorisent pas les mêmes dimensions de leur identité numérique professionnelle, notamment dans la formalisation de leur statut professionnel. Par exemple, si les élèves des grandes écoles mettent en avant l'étiquette valorisante de leur institution, les étudiants de l'université, eux, valorisent plutôt leur champ de compétences. C'est donc l'enchevêtrement de trois facteurs explicatifs, technique, social et individuel qui permet de rendre compte de ces pratiques de réseautage en ligne et de la façon dont elles contribuent à l'élaboration d'une identité numérique professionnelle.

À présent, nous allons, en nous appuyant sur une enquête préalablement conduite auprès de jeunes de 16 à 24 ans de la région PACA, mettre en évidence la nature des usages informationnels et communicationnels mis en œuvre lors de la construction de leur trajectoire professionnelle et voir si ces derniers révèlent l'existence d'une identité numérique professionnelle. 
Maude PÉLISSIER

\section{L'enquête et ses résultats}

\subsection{Le cadre méthodologique}

Les résultats de l'enquête présentés ici sont issus d'une étude quantitative réalisée dans le cadre de l'Observatoire des TIC (baromètre 2013 du Conseil régional) visant à révéler comment les jeunes de 16 à 24 ans mobilisent les ressources numériques dans un contexte d'insertion professionnelle. Elle n'avait donc pas pour objectif d'interroger directement la notion d'identité numérique professionnelle. Ce n'est qu'a posteriori que cette question a émergé, ce qui explique, comme on pourra le voir, les raisons pour lesquelles cette enquête n'est pas entièrement calibrée pour répondre parfaitement au cadre théorique précédemment évoqué. Toutefois, certains des résultats nous semblaient importants pour éclairer ce concept encore balbutiant.

Cette enquête comporte un ensemble de questions réparties en trois thématiques. La première porte sur une étude des usages numériques des jeunes dans leur quotidien, la deuxième sur une évaluation de leur niveau de culture informationnelle et communicationnelle au travers d'un questionnaire de dix questions (degré de connaissance de l'environnement numérique) et, enfin, la dernière révèle les usages numériques des jeunes dans le contexte plus circonscrit de la construction de leur trajectoire professionnelle. Le choix a été fait d'administrer le questionnaire en ligne sur un site Internet dédié (www.tic1624.fr) afin d'attirer l'attention des jeunes et de les inciter à y répondre. Le questionnaire a été activé du 28 mars au $1^{\mathrm{er}}$ septembre 2013. Pour susciter l'intérêt des jeunes et leur donner envie de participer et de partager le questionnaire à leurs proches, nous l'avons présenté sous une forme ludique en proposant aux répondants d'obtenir leur profil numérique à la fin. Sept profils avaient été définis (geek, noob, curieux, averti, résigné, analogique, rebelle) pour qualifier les questionnés en fonction de leurs réponses quant à leur intensité d'usage, leur degré de connaissances et leur confiance envers l'environnement numérique. Enfin, nous avons opté pour une démarche de community management afin de réunir le plus grand nombre de réponses possibles, en jouant sur la viralité de l'enquête. La population mère comportait 587392 individus (dernier recensement INSEE, 2009). À la fin de la période de mise en ligne, nous avions obtenu 743 participations. Ce premier échantillon a ensuite été redressé (489 répondants) afin de n'avoir que des répondants de la région PACA ayant entre 16 et 24 ans (exigence du commanditaire). Les variables de contrôle retenues sont le 
La construction d'une identité numérique professionnelle chez les « digitales natives » : fiction ou réalité ?

sexe et le statut (actifs, étudiants, lycéens). Malheureusement, le faible nombre de répondants dans la catégorie actifs ne nous a pas permis de construire un échantillon représentatif crédible. Nous avons donc choisi de travailler sur l'échantillon de 489 répondants, lequel intègre une surreprésentation des étudiants et une sous-représentation des actifs. Nous avons tenu compte de ce biais lors des calculs effectués, puis lors de l'analyse des résultats.

\subsection{Le cadre opératoire: les indicateurs proposés}

Dans la première partie, nous avons montré que les jeunes se retrouvent dans un cadre technique économique et institutionnel qui les incite à se forger une identité professionnelle numérique. Pour autant, les jeunes prennent-ils la mesure de ce changement et mettent-ils en œuvre de telles stratégies ? L'objet de l'étude empirique présentée ici est de mettre en perspective les usages informationnels et communicationnels mis en place par les jeunes dans la construction de leur trajectoire professionnelle et d'évaluer si ceux-ci témoignent d'une stratégie de mise en œuvre d'une identité numérique professionnelle.

La première étape de l'enquête a consisté à construire des indicateurs permettant d'appréhender, d'un point de vue empirique, l'identité numérique professionnelle. Trois indicateurs ont été définis. Le premier porte sur les pratiques informationnelles. Choisir son orientation professionnelle, pour un lycéen ou un étudiant, chercher un stage ou un premier emploi, pour un étudiant, se traduit par des pratiques de recherche d'informations, aussi bien formelles qu'informelles, dans l'univers numérique comme en dehors. Dans cette perspective, avoir la volonté de se construire une identité numérique professionnelle devrait se manifester, à un premier niveau, par une mobilisation importante des ressources informationnelles présentes dans l'environnement numérique révélant, en particulier, des réseaux sociaux numériques professionnels.

Les deux autres indicateurs portent sur les pratiques communicationnelles accompagnant la construction de la trajectoire professionnelle qui témoigne d'une stratégie de mise en œuvre d'une identité numérique professionnelle. D'une part, la stratégie de mise en visibilité du curriculum vitæ nous a semblé un indice déterminant. Celui-ci, qui constituait naguère le passeport indispensable pour franchir les frontières du monde du travail, connaît aujourd'hui des évolutions notoires tant sur la forme que dans ses modalités de diffusion, comme nous l'avons évoqué. Nous faisons ici l'hypothèse que, au vu 


\section{Maude PÉLISSIER}

de l'intensité des usages numériques avérée de cette tranche d'âge, le curriculum vitæ papier n'est plus la forme dominante d'expression de son identité numérique professionnelle. D'autre part, nous faisons l'hypothèse que les jeunes transfèrent l'intensité et leur expertise des RSN du type Facebook vers les RSN professionnels lorsqu'il s'agit d'élaborer leur trajectoire professionnelle. Ainsi, cela signifie non seulement être inscrit sur ces réseaux, mais aussi en avoir un usage régulier, témoignant de besoins propres à leur statut (étudiant ou actif).

\subsection{Présentation des résultats}

3.3.1. Premier indicateur: l'utilisation des ressources numériques dans les choix d'orientation professionnelle

Le premier indicateur a été construit à partir des réponses données à deux questions. La première avait pour but de mettre en évidence la nature des ressources informationnelles privilégiées par les lycéens et les étudiants dans leurs choix en matière d'orientation professionnelle (Figure 1). La seconde visait à évaluer les procédures de recherche d'informations mises en œuvre pour leur insertion professionnelle afin d'évaluer à la fois leur niveau d'expertise et la place des RSN professionnels (Figure 2).

ou écoles...)

Figure 1. Les ressources informationnelles privilégiées par les lycées et les étudiants dans leur trajectoire d'orientation professionnelle (question à choix multiples)

On peut remarquer que les pratiques informationnelles des jeunes interrogés ne privilégient pas de façon évidente les ressources informationnelles du monde numérique (consultation de sites Web spécialisés, forums, blogues, sites d'école) et que les ressources du monde physique (conseil des parents et des amis, discussions avec les enseignants, salons) ont encore un rôle prépondérant, notamment du côté des lycéens. On est donc plutôt en présence d'une cohabitation entre ces différents types de ressources.

Concernant le degré d'expertise associé aux pratiques informationnelles accompagnant la recherche d'un stage ou d'un emploi dans l'environnement numérique, deux constats peuvent être dressés (Figure 2). Premièrement, plus les jeunes entrent dans la vie active, plus ils vont chercher des ressources informationnelles en mobilisant tous les outils à leur disposition pour optimiser leurs recherches (signets, alertes). Ils ont une stratégie de recherche d'informations « multicanal », destinée à 
La construction d'une identité numérique professionnelle chez les « digitales natives » : fiction ou réalité ?

optimiser la qualité de leurs résultats. Deuxièmement, l'usage des RSN professionnels est une pratique inexistante chez les lycéens et encore minoritaire chez les étudiants et les jeunes actifs.

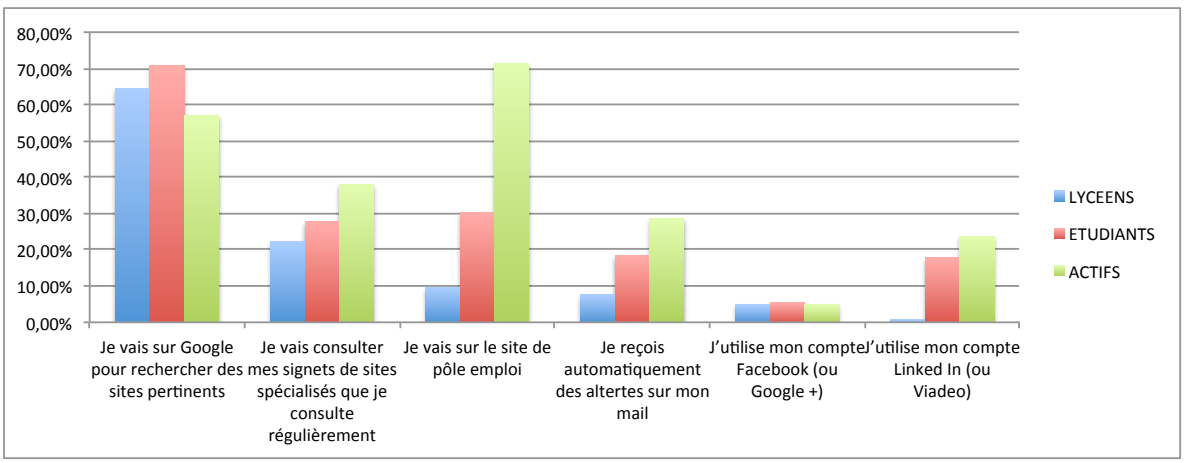

Figure 2. Les procédures de recherche d'informations sur le Web pour trouver un stage ou un emploi (question à choix multiples)

\subsubsection{Deuxième indicateur : la mise en visibilité du} curriculum vitæ

La première question retenue pour cet indicateur portait sur les modalités de diffusion du curriculum vitæ parmi les étudiants et les actifs (Figure 3 ). Les résultats obtenus ne reflètent pas l'hypothèse de travail émise : sa mise en visibilité se traduit, pour ce qui est de ses modalités de diffusion, par une "cohabitation papier/numérique ". La diffusion par voie postale n'a pas encore complètement disparu, comme on aurait pu le supposer dans cette tranche d'âge, même si elle est moindre chez les jeunes actifs qui privilégient en deuxième choix les sites spécialisés. On peut s'interroger sur les raisons d'une telle hybridation dans les pratiques de publicisation du curriculum vitæ à la fois papier et numérique. Deux hypothèses peuvent être émises : soit il s'agit de personnes qui ont une faible acculturation au numérique, soit cela est dû à certains secteurs d'emploi qui restent encore sur des modes d'appariement traditionnels. 
Maude PÉLISSIER

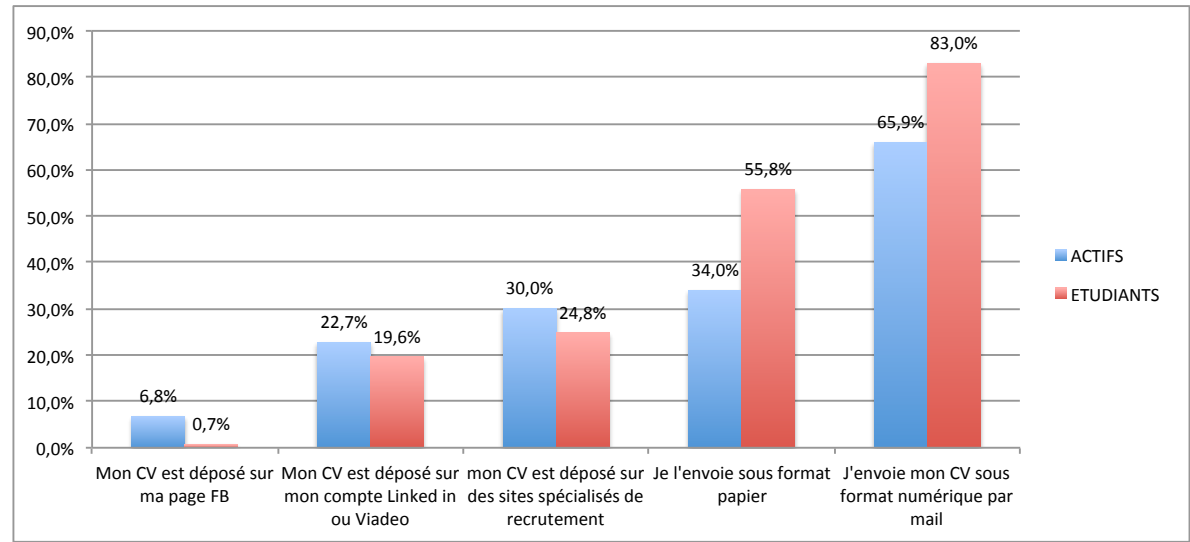

Figure 3. Modalités de diffusion du curriculum vitæ parmi les étudiants et les actifs

Ensuite, on peut constater qu'une proportion assez équivalente (autour de $20 \%$ ) des jeunes interrogés a déposé son curriculum vitæ sur un RSN professionnel et que quasiment aucun d'entre eux ne l'a fait sur sa page Facebook. Les jeunes opèrent ainsi un cloisonnement dans les usages qu'ils font des RSN. Facebook est un RSN orienté vers des pratiques communicationnelles dans un contexte ludique et de divertissement, et non un dispositif de communication pour la construction de leur trajectoire professionnelle. Ce résultat est indirectement corroboré par le fait que, sur ces réseaux socionumériques traditionnels, les jeunes ont un nombre très important d' " amis", mais que les "relations professionnelles " n'en font quasiment pas partie. Celles-ci représentent moins de $5 \%$ des « amis » pour les étudiants et les actifs (Figure 4).

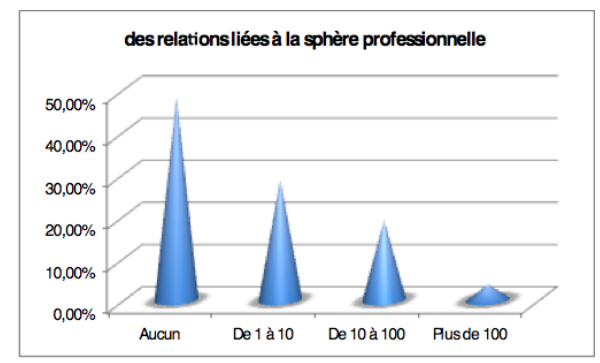

Figure 4. Nombre de relations professionnelles parmi les « amis » sur le compte Facebook des jeunes de 16 à 24 ans 
La construction d'une identité numérique professionnelle chez les « digitales natives » : fiction ou réalité ?

3.3.3.Troisième indicateur: présence sur les réseaux sociaux numériques professionnels

Cet indicateur a été élaboré à partir de deux questions : l'une portant sur le fait de savoir si les jeunes interrogés avaient un compte sur un RSN professionnel; la seconde, sur leur intensité de publication sur ces mêmes réseaux.

Le premier constat issu des résultats de l'enquête est une présence relativement faible sur les RSN professionnels (Figure 5). En effet, si 89,3\% des jeunes ont un compte sur le RSN Facebook, seulement $25 \%$ d'entre eux en ont un sur un RSP (Viadeo et LinkedIn). On note une appropriation croissante en fonction du statut : $6,6 \%$ des lycéens, $30 \%$ des étudiants, $50 \%$ des actifs.

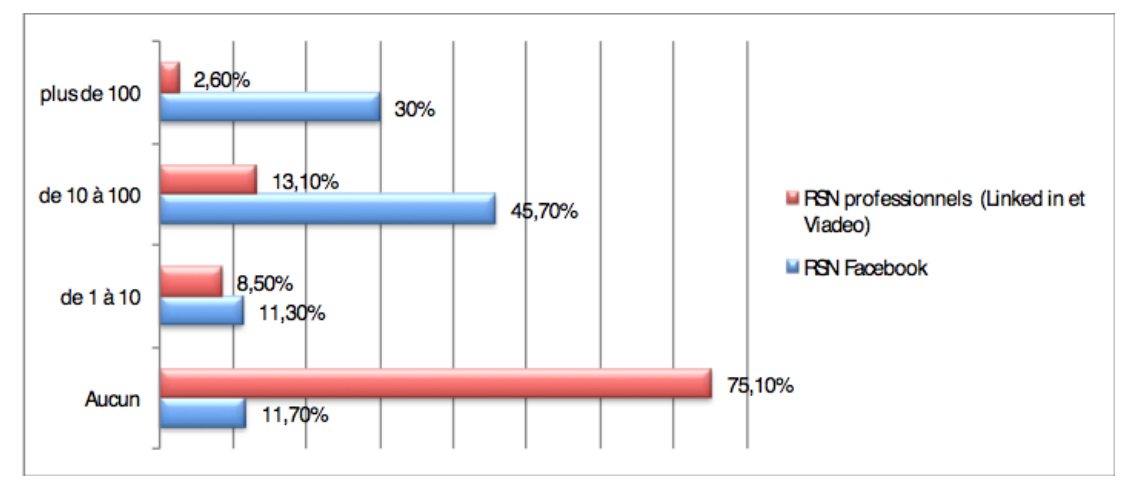

Figure 5. Nombre de contacts sur les réseaux sociaux professionnels chez les jeunes de 16 à 24 ans

Le second constat révèle une fréquence de publication très faible, et ce, quel que soit leur statut : $86,9 \%$ ne publient jamais sur Linkedln et 82,7\%, jamais sur Viadeo (Figure 6). Ce résultat montre que les $25 \%$ des jeunes qui ont un compte sur un RSN professionnel n'en font un usage que très épisodique, voire nul. Ce résultat varie légèrement suivant leur statut (Figure 7) et indique que, si les RSN professionnels sont étrangers aux lycéens, ils constituent un dispositif beaucoup plus utilisé par les jeunes actifs. 


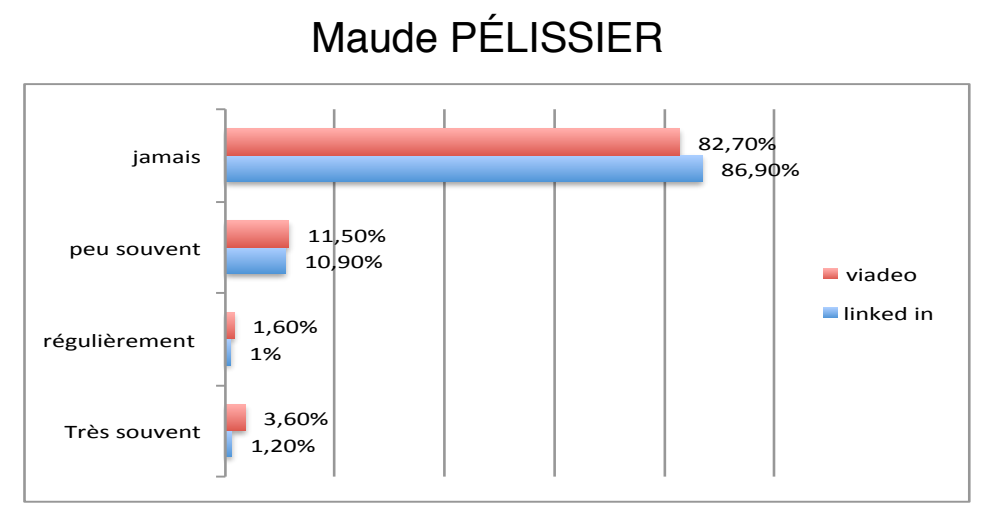

Figure 6. Fréquence de publication sur les réseaux sociaux professionnels chez les jeunes de 16 à 24 ans (Viadeo, LinkedIn)

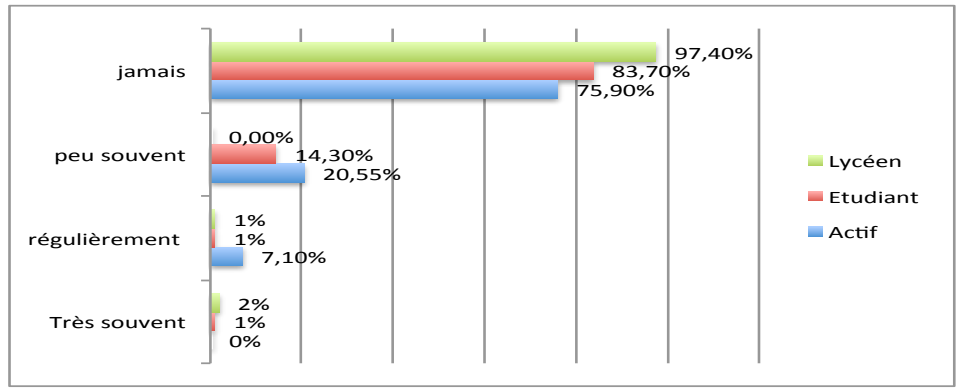

Figure 7. Fréquence de publication sur les réseaux sociaux professionnels suivant le statut

Par ailleurs, le tri croisé entre le nombre de contacts et la fréquence de publication ne montre pas que ceux qui publient le plus sont ceux qui ont le plus de contacts. De même, celui entre la fréquence de publication sur FB et sur Linkedln ne présente pas de dépendance significative : ceux qui publient le plus sur FB ne sont pas ceux qui publient le plus (ou le moins) sur Linkedln. Le résultat est le même avec le réseau Viadeo.

Une dernière question (fermée à choix unique) a été posée aux jeunes à propos de leur perception des réseaux professionnels numériques. La majorité $(57,6 \%)$ considère ne pas avoir d'avis sur la question et seulement un tiers $(33,3 \%)$ pense que "c'est un moyen de rencontrer des experts dans son domaine professionnel ॥ (Figure 8). Cette dernière proportion correspond globalement aux jeunes qui utilisent ces réseaux numériques. Il y a donc une cohérence entre leur présence sur ces réseaux et l'utilité qu'ils espèrent en retirer, même s'ils en font un usage très épisodique. 
La construction d'une identité numérique professionnelle chez les « digitales natives » : fiction ou réalité ?

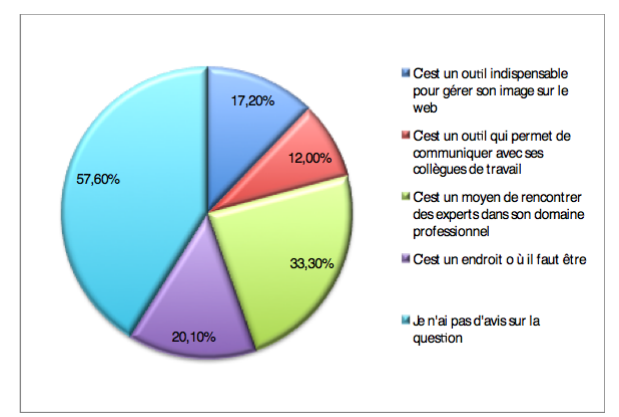

Figure 8. Perception des réseaux professionnels numériques chez les jeunes

Enfin, notons que très peu perçoivent que leur présence sur les réseaux sociaux a une incidence sur leur réputation (seulement $17,2 \%$ d'entre eux pensent que "c'est un outil indispensable pour gérer [leur] image sur le Web »).

\section{Conclusion}

Partant des travaux de Dominique Cardon $(2008,2009)$ sur l'identité numérique, nous avons défini conceptuellement l'identité numérique professionnelle comme une dynamique d'extériorisation de son moi professionnel dans des signes rendus visibles sur les différents réseaux socionumériques professionnels.

Le design de ces réseaux incite l'usager à adopter un comportement rationnel réflexif consistant à optimiser la mise en visibilité de sa trajectoire professionnelle au travers de la mise en œuvre de pratiques de réseautage. Ces RSN professionnels traduisent, d'un point de vue technologique, le discours de leurs concepteurs, qui défendent l'idée qu'ils constituent des leviers indispensables dans la réussite de la trajectoire professionnelle en offrant à chacun la possibilité de se constituer un capital social élaboré à partir de la construction d'un réseau social. Dans la même lignée, nous avons montré que la perméabilité des différents espaces du Web ne permettant plus de dissocier les espaces publics des espaces privés, les espaces de loisirs des espaces professionnels, conduit aussi l'usager à maîtriser son identité numérique au risque d'avoir à en payer des conséquences graves pour sa réputation professionnelle. Dans l'environnement numérique, la trajectoire professionnelle est un capital qu'il faut savoir non seulement valoriser, telle une image de marque, mais aussi contrôler à une échelle plus globale. 
Au travers d'une enquête quantitative visant à révéler comment les jeunes de 16 à 24 ans utilisent les ressources numériques dans leurs trajectoires d'insertion professionnelle, nous avons tiré quelques enseignements éclairants sur la question de l'identité numérique professionnelle en révélant des écarts en matière d'usage par rapport aux espaces de prescription précédemment évoqués.

L'identité numérique professionnelle est encore un processus en gestation chez les jeunes de 16 à 24 ans, processus qui ne renvoie manifestement pas à une logique de rationalité calculatoire. II ne s'agit pas d'une fiction, des indices attestant d'une évolution de leurs pratiques, mais, pour autant, on ne peut pas dire qu'il s'agit d'une réalité nouvelle partagée par l'ensemble de cette tranche d'âge. Le premier indicateur, défini comme la capacité à mobiliser des ressources et des dispositifs informationnels disponibles sur le Web dans la construction de leur trajectoire professionnelle, montre que les dispositifs numériques occupent désormais une place importante et accompagnent de façon complémentaire les autres sources potentielles des jeunes pour prendre leur décision. Le deuxième indicateur, relatif à la stratégie communicationnelle numérique mise en œuvre, a révélé une capacité réelle à mobiliser des dispositifs numériques tout en maintenant encore les modalités de diffusion propres à la culture de l'imprimé (en particulier concernant les modalités de diffusion du curriculum vitæ). On a également pu constater que seule une minorité est membre d'un réseau social numérique professionnel, même si les jeunes actifs sont plus prompts à les utiliser que leurs cadets (pratique inexistante chez les lycéens). À l'inverse du réseau social Facebook, qui constitue pour les jeunes interrogés un espace de communication interpersonnelle intimement lié à leur vie quotidienne hors travail, les réseaux sociaux professionnels numériques comme Linkedln ou Viadeo n'ont pas encore une véritable popularité pour se positionner comme des dispositifs permettant d'appuyer la mise en œuvre d'une identité numérique professionnelle. De surcroît, une infime minorité en fait un usage régulier.

Qu'est-ce qui explique que ces plateformes relationnelles professionnelles sont encore si éloignées de leurs usages numériques ? En effet, il apparaît clairement un fossé entre leur dire (la majorité affirmant que les RSN professionnels sont des dispositifs clés pour gérer leur image sur le Web) et leur faire, révélant des usages non stratégiques dans l'appropriation de ces plateformes relationnelles professionnelles. Si notre enquête a permis de mettre en évidence cet écart comme résultat intéressant, elle ne nous a pas permis d'examiner plus 
La construction d'une identité numérique professionnelle chez les « digitales natives » : fiction ou réalité ?

finement les raisons d'un tel écart. II aurait pour cela fallu compléter notre étude empirique par une analyse qualitative, offrant la possibilité d'interroger plus finement les jeunes sur la base d'une catégorisation ex post et d'entretiens semi-directifs ciblés.

Néanmoins, nous proposons d'esquisser des pistes éventuelles pour expliquer un tel écart, pouvant faire l'objet d'un travail d'investigation futur. Premièrement, l'environnement numérique reste encore et avant tout pour les jeunes un univers ludique de divertissement et de culture. Cette segmentation traduit le fait que le contexte socioéconomique, décrit dans la première partie, n'a pas encore une influence décisive sur les pratiques communicationnelles et informationnelles des jeunes dans l'environnement numérique. Cette hypothèse nous semble être particulièrement plausible pour les jeunes lycéens qui ne sont pas inscrits sur ces plateformes.

Deuxièmement, on peut supposer que, si les jeunes ne font pas usage de ces réseaux, c'est aussi parce qu'ils n'ont qu'une connaissance relativement limitée de leurs potentialités et de leur efficacité dans la construction de leur trajectoire professionnelle. Cela renvoie à la question de la maîtrise d'une culture informationnelle et communicationnelle (Le Deuff, 2011) comme un levier désormais essentiel dans la construction de la trajectoire professionnelle. Rappelons qu'un certain nombre d'études montrent que cette culture chez les "digital natives » adolescents est loin d'être distribuée de façon homogène (Mercklé et Octobre, 2012) et que ce non-usage constaté témoigne souvent d'une incapacité à mobiliser cette culture dans une optique d'amélioration de son bien-être socioéconomique, ce qui se traduit par de nouvelles formes d'inégalités (Granjon, 2011). C'est à une conclusion similaire à laquelle aboutit l'étude empirique réalisée dans les missions locales pour l'emploi auprès d'un public de jeunes en difficulté d'insertion : " Au-delà du parcours professionnel à proprement parler, aider les jeunes à utiliser pleinement le potentiel des TIC, c'est également leur permettre de mieux s'insérer et de s'ouvrir à de nouveaux horizons » (Davenel, 2015, p. 23).

Finalement, une dernière hypothèse, complémentaire, peut être émise pour comprendre le non-usage observé des RSN professionnels Linkedln et Viadeo. Ces plateformes ne constituent pas à proprement parler des outils visant à l'employabilité qui, rappelons-le, n'est pas leur mission première. Les chiffres attestent d'ailleurs d'une attractivité globale relativement faible de ces plateformes auprès des jeunes (Linkedln prétend réunir entre 7 et 8 millions d'étudiants sur environ 300 millions de membres). Ce sont avant tout des dispositifs de mise en relation et de gestion de carrière faisant 
office de carnet d'adresses pour des personnes en situation d'emploi. Être sur ces réseaux présente cependant un avantage indéniable pour les jeunes, celui d'accroître leur visibilité numérique grâce au très bon référencement de ces plateformes relationnelles. C'est peut-être la raison pour laquelle on y trouve, parmi les jeunes, un si grand nombre d'usagers passifs. Ainsi, le non-usage stratégique observé peut aussi être interprété comme le résultat d'une stratégie identitaire professionnelle face à des recruteurs potentiels qui agrègent par le biais d'Internet les informations disponibles sur les demandeurs d'emploi (Larroche et Bérard, 2014).

Or on peut aussi considérer le problème autrement, en supposant que les jeunes ne sont pas incités à adopter une stratégie de construction de leur identité numérique professionnelle, car ils ne le perçoivent pas comme une étape majeure. II est vrai qu'à ce jour, certains secteurs professionnels (communication, marketing, TIC) sont plus prompts à mobiliser ce type de dispositif et que, pour de nombreux autres, l'appariement sur le marché du travail ne s'opère pas au travers de la médiation de ces dispositifs numériques. Pour sélectionner un futur communicant, il peut paraître logique qu'un employeur évalue sa capacité à savoir se mettre en valeur professionnellement. Mais cela ne peut être valable pour tous les recruteurs et toutes les professions.

\section{RÉFÉRENCES BIBLIOGRAPHIQUES}

ALLARD L., 2007, « Émergence de cultures expressives, d'Internet au mobile », MédiaMorphoses, n² 21 pp. 1925.

ALLOING C. et PIERRE J., 2012, « Construire un cadre d'analyse avec les SIC pour comprendre les pratiques et les enjeux de la réputation en ligne (des individus et des organisations) », <https://hal.archives-ouvertes.fr/hal$00704007>$, dernière consultation le 2 février 2017.

BEN AMOR S. et GRANGET L., 2011, " L'Identité numérique : de la construction au suicide en 52 minutes ", Les Cahiers du numérique, vol. 7, pp. 103-115.

BEVORT B. et BRÉDA I., 2003, « Les jeunes et Internet: représentations, usages et appropriations ", rapport du Centre de liaison de l'enseignement et des médias de l'information (Clémi). 
La construction d'une identité numérique professionnelle chez les « digitales natives » : fiction ou réalité ?

CARDON D., 2009, « L'Identité comme stratégie relationnelle », Hermès, $n^{\circ} 53$, pp. 61-66.

CARDON D., 2008, « Le Design de la visibilité : un essai de cartographie du Web 2.0 », Réseaux, n¹52, pp. 93-137.

COUTANT A. et STENGER A., 2010, « Processus identitaire et ordre de l'interaction sur les réseaux socionumériques », Les enjeux de l'information et de la communication, vol. 1, pp. 45-64.

COUTANT A., 2015, " Les approches sociotechniques dans la sociologie des usages en SIC ॥, Revue française des sciences de l'information et de la communication, vol. 6, <http://rfsic.revues.org/1271>, dernière consultation le 7 février 2017.

DAUPHIN F., 2012. «Culture et pratiques numériques juvéniles : quels usages pour quelles compétences ? ", vol. $7, \mathrm{n}^{\circ} 17$, <https://questionsvives.revues.org/988>, dernière consultation le 2 février 2017.

DAVENEL Y.-M., 2015, " Les pratiques numériques des jeunes en insertion socioprofessionnelle », Les études connexions solidaires, <http://emmaus-connect.org/wpcontent/uploads/2015/06/Etude-Emmaus-Connect-Lespratiques-num\%C3\%A9riques-des-jeunes-en-insertionsocioprofessionnelles-Mai-2015.pdf $>$, dernière consultation le 2 février 2017.

DOMENGET, J.-C. (2013, mars), « Construire son e-réputation sur Twitter: les pratiques instrumentalisées de professionnels de la visibilité ", Colloque international « E-réputation et traces numériques. Dimensions instrumentales et enjeux de société ", Toulouse.

DOUEIHI M., 2008, La Grande Conversion numérique, Paris, Seuil.

ERTZSCHEID O., 2009, « L'Homme, un document comme les autres », Hermès, vol. 53, pp. 33-40.

GEORGES F., 2009, « Représentation de soi et identité numérique », Réseaux, n 154, pp. 165-193.

GRANJON F., 2011, « Fracture numérique », Communications, $\mathrm{n}^{\circ}$ 88, pp. 67-75.

JOUËT J., 1993, « Pratiques de communication et figures de médiation », Réseaux, n60, vol. 11, pp. 99-120.

LARROCHE V. et BÉRARD J., 2014, « Stratéqies identitaires professionnelles à l'université : les premiers pas vers l'employabilité numérique », Enseignement, préservation 
et diffusion des identités numériques, pp. 215-241, <https://hal.archives-ouvertes.fr/hal-00808715>, dernière consultation le 2 février 2017.

LE DEUFF O., 2011, La Formation aux cultures numériques, une nouvelle pédagogique pour une nouvelle culture de l'information à l'ère du numérique, Limoges, FYP Éditions.

LELONG M., 2013, « II n'y a pas d'âge pour le réseautage », $M$ le mag (France), 23 septembre 2013, $<$ www.lemonde.fr/m-actu/article/2013/09/21/il-n-y-a-pasd-age-pour-le-reseautage_3480535 4497186.html>, dernière consultation le 2 février $20 \overline{17}$.

LIVINGSTONE S., ÓLAFSSON K. et STAKSRUD E., 2011, "Social Networking, Aqe and Privacy », EU Kids Online, $<$ www.Ise.ac.uk/media@Ise/research/EUKidsOnline/EU $\% 20$ Kids\%2011\%20(2009-

11)/EUKidsOnlinelIReports/ShortSNS.pdf>, dernière consultation le 2 février 2017.

LONGO M., 2011, « Transitions des jeunes vers la vie adulte: processus d'insertion et rapports à la vie professionnelle. Perspectives pour les programmes d'action », Rapport d'étude de l'Institut national de la jeunesse et de l'éducation populaire (INJEP), $<w w w . i n j e p . f r / s i t e s / d e f a u l t / f i l e s / d o c u m e n t s / T r a n s i t i o n s-$ des-jeunes-vers-la-vie-adulte.pdf $>$, dernière consultation le 2 février 2017.

MERCKLÉ P., 2011, Sociologie des réseaux sociaux, Paris, La Découverte.

MERCKLÉ O et OCTOBRE S., 2012, « La stratification sociale des pratiques numériques des adolescents ॥, Reset, $\mathrm{n}^{\circ} 1,<$ https://reset.revues.org/129>, dernière consultation le 2 février 2017.

MERZEAU L., 2009, « Présence numérique : les médiations de l'identité », Les enjeux de l'information et de la communication, $\mathrm{n}^{\circ} 1$, pp. 79-91.

MÉSENGEAU J., 2012, « Figures du "réseautage en ligne” sur les réseaux socionumériques professionnels : le cas d'un groupe d'anciens sur Linkedln » (thèse de doctorat), Université Rennes 2.

MÉSENGEAU J. et POVÉDA A., 2013, « Les réseaux sociaux professionnels en ligne. Vers une polyinstitutionnalisation du marché du travail ? ", Revue européenne du droit social, vol. 19, n 2, pp. 119-136. 
La construction d'une identité numérique professionnelle chez les « digitales natives » : fiction ou réalité ?

ORIGGI G., 2007, « Un certain regard : pour une épistémologie de la réputation ", Communications, $n^{\circ}$ 93, pp. 101-120.

PÉLISSIER M. et PÉROCHEAU G., 2013, « Cultures numériques et trajectoires professionnelles chez les 1624 ans ", Enquête ObTIC (Observatoire des technologies de l'information et de la communication en région $\mathrm{PACA}$ ), <http://emergencesnumeriques.regionpaca.fr/fileadmin/Economie_Creative Groupe de travail/OBTIC/jeunes-tic/ENQUETEjeunes TIC-Ōbtic-2013.pdf>, dernière consultation le 2 février 2017.

PIERRE J., 2011, « Génétique de l'identité numérique : sources et enjeux des processus associés à l'identité numérique », Les Cahiers du numérique, vol. 7, pp. 1529.

PRENSKY M., 2001, « Digital Natives, Digital Immigrants Part $1 »$, On the Horizon, vol. 9, $\mathrm{n}^{\circ} 5$, $<$ www.emeraldinsight.com/doi/pdfplus/10.1108/1074812 0110424816>, dernière consultation le 2 février 2017.

STOYCHEFF, E., 2016, « Under Surveillance: Examining Facebook's Spiral of Silence Effects in the Wake of NSA Internet Monitoring », Journalism \& Mass

Communication Quarterly, vol. 93, n² 2, p. 296-311. 